\title{
Macrophage-derived IL-10 mediates mucosal repair by epithelial WISP-1 signaling
}

\author{
Miguel Quiros, ${ }^{1}$ Hikaru Nishio, ${ }^{2}$ Philipp A. Neumann, ${ }^{3}$ Dorothee Siuda, ${ }^{1}$ Jennifer C. Brazil, ${ }^{1}$ Veronica Azcutia, ${ }^{1}$ Roland Hilgarth, ${ }^{1}$ \\ Monique N. O'Leary, ${ }^{1}$ Vicky Garcia-Hernandez, ${ }^{1}$ Giovanna Leoni, ${ }^{4}$ Mingli Feng, ${ }^{1}$ Gabriela Bernal, ${ }^{1}$ Holly Williams, ${ }^{1}$ Priya H. Dedhia, ${ }^{5}$ \\ Christian Gerner-Smidt, ${ }^{2}$ Jason Spence, ${ }^{5}$ Charles A. Parkos, ${ }^{1}$ Timothy L. Denning, ${ }^{6}$ and Asma Nusrat ${ }^{1}$
}

'Department of Pathology, University of Michigan, Ann Arbor, Michigan, USA. ²Department of Pathology, Emory University, Atlanta, Georgia, USA. ${ }^{3}$ Department of Surgery, Klinikum rechts der Isar, Technische Universität München, Munich, Germany. ${ }^{4}$ Institute for Cardiovascular Prevention (IPEK), Ludwig-Maximilian University (LMU) Munich, Munich, Germany. ${ }^{5}$ Department of Internal Medicine and Department of Cell and Developmental Biology, University of Michigan, Ann Arbor, Michigan, USA. ${ }^{6}$ Center for Inflammation, Immunity and Infection, Institute for Biomedical Sciences, Ceorgia State University, Atlanta, Georgia, USA

In response to injury, epithelial cells migrate and proliferate to cover denuded mucosal surfaces and repair the barrier defect. This process is orchestrated by dynamic crosstalk between immune cells and the epithelium; however, the mechanisms involved remain incompletely understood. Here, we report that IL-10 was rapidly induced following intestinal mucosal injury and was required for optimal intestinal mucosal wound closure. Conditional deletion of IL-10 specifically in CD11c-expressing cells in vivo implicated macrophages as a critical innate immune contributor to IL-10-induced wound closure. Consistent with these findings, wound closure in T cell- and B cell-deficient Rag $1^{-/-}$mice was unimpaired, demonstrating that adaptive immune cells are not absolutely required for this process. Further, following mucosal injury, macrophage-derived IL-10 resulted in epithelial cAMP response element-binding protein (CREB) activation and subsequent synthesis and secretion of the pro-repair WNT1-inducible signaling protein 1 (WISP-1). WISP-1 induced epithelial cell proliferation and wound closure by activating epithelial pro-proliferative pathways. These findings define the involvement of macrophages in regulating an IL-10/CREB/WISP-1 signaling axis, with broad implications in linking innate immune activation to mucosal wound repair.

\section{Introduction}

The gastrointestinal epithelium forms a selective barrier that plays a pivotal role in separating the microbe-rich luminal contents from underlying tissue compartments, while also regulating the absorption of nutrients. Epithelial damage associated with disruption of the intestinal mucosal barrier occurs following mechanical injury and is a hallmark of several pathologic states including inflammatory bowel disease (IBD) (1). Efficient repair of this barrier is critical for suppressing mucosal inflammation and reestablishing intestinal homeostasis. Epithelial wound closure is orchestrated by a series of events involving the epithelium itself as well immune cells from both the innate and adaptive immune systems that are recruited into the wound. After an initial recruitment of neutrophils, there is an influx of macrophages that reside near the epithelium. Macrophages have been reported to secrete anti-inflammatory and pro-resolution mediators such as TGF- $\beta$, VEGF, metalloproteinases, and IL-10 (2).

The anti-inflammatory cytokine IL-10 has been reported to regulate intestinal mucosal homeostasis (3). Mice lacking IL-10 are prone to developing spontaneous enterocolitis within 4 to 8 weeks of age (4). Intestinal mucosal macrophages are the major source of IL-10 in the intestinal mucosa (5). While the antiinflammatory effects of IL-10 on immune cells and mucosal homeostasis have been well studied, the role of IL-10 in orchestrating wound repair

Authorship note: M. Quiros, H. Nishio, and P.A. Neumann contributed equally to this work. Conflict of interest: The authors have declared that no conflict of interest exists. Submitted: August 18, 2016; Accepted: June 27, 2017.

Reference information: J Clin Invest. 2017;127(9):3510-3520.

https://doi.org/10.1172/JCI90229. remains less clear. Here, we report the pro-repair properties of IL-10 and identify the mechanism by which IL-10 promotes epithelial cell proliferation and wound repair in the intestine. We show that IL-10 promotes intestinal epithelial wound healing through the activation of CREB signaling, with subsequent synthesis and secretion of the WNT1-inducible signaling protein 1 (WISP-1). WISP- 1 is a member of the connective tissue growth factor (CTGF) family and has been increasingly recognized for its roles in facilitating repair (6). Our study identifies a link between the antiinflammatory cytokine IL-10 and WISP-1, which serve to promote epithelial wound repair and restore mucosal homeostasis.

\section{Results}

IL-10 expression is increased in resealing intestinal mucosa. IL-10 suppresses immune cell pro-inflammatory responses and plays an important role in maintaining homeostasis (3). However, the role of IL-10 in promoting mucosal repair is not well defined. To explore the pro-repair properties of IL-10, we performed a kinetic analysis of IL-10 effects on epithelial wound closure in vitro using the intestinal epithelial cell line SKCO-15. As shown in Figure 1A, exposure to IL-10 increased wound repair within 12 hours, and this response was further enhanced after 24 hours $(P<0.01,12$ h; $P<0.001,24$ h). These data were consistent with IL-10 receptor subunit $\alpha$ (IL-10R $\alpha$ ) expression by SKCO-15 cells (Figure 1B). To determine the specificity of IL-10 effects on epithelial wound repair, IL-10R $\alpha$ was downregulated using an siRNA approach. As shown in Figure 1C, IL-10R $\alpha$ knockdown inhibited the IL-10induced increase in wound closure following IL-10 treatment. Since IL-10 increased in vitro epithelial wound repair, we evalu- 
A
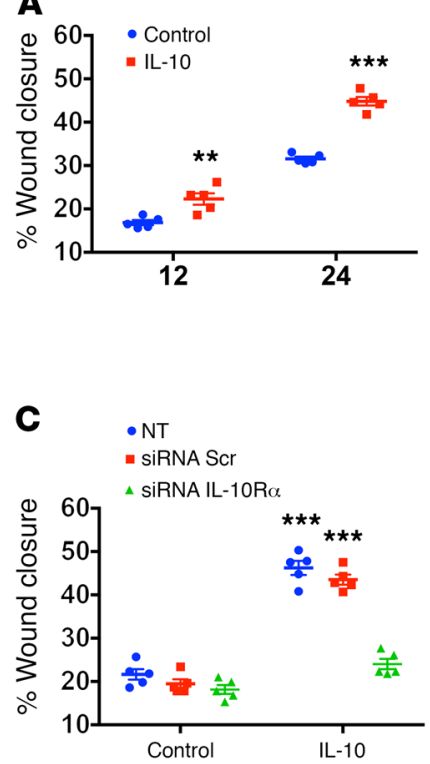

E

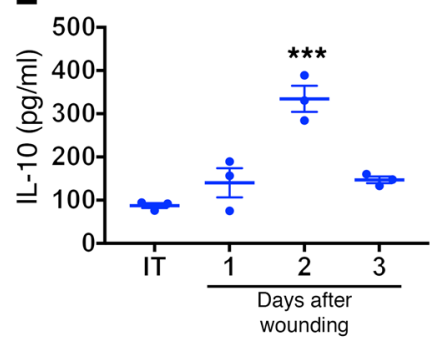

B

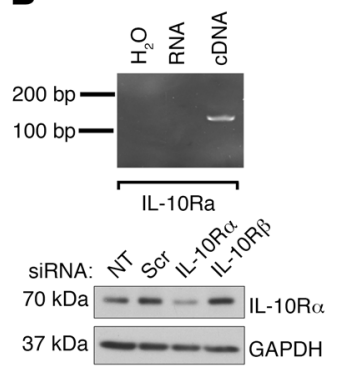

D

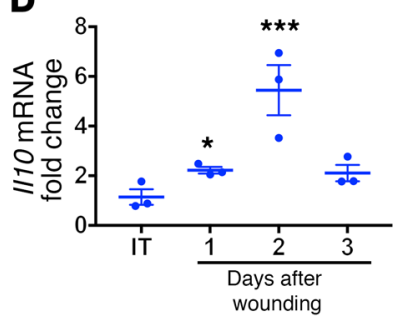

$\mathbf{F}$

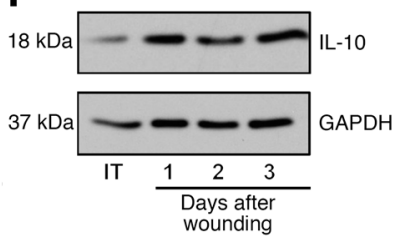

Figure 1. IL-10 is synthesized and released as a response to intestinal mucosal injury. (A) Scratch wound-healing assay using IEC monolayers. rhIL-10 was added to wounded IECs, and wound widths were determined 0,12 , and 24 hours after injury ${ }^{* *} P<0.01$ and ${ }^{* *} P<0.001, n=5$, mean \pm SEM). (B) IEC expression of IL-10R $\alpha$ was analyzed by GPCR and Western blotting. (C) Scratch wound-healing assay in IEC monolayers. Cells were transfected or not transfected with either a scramble siRNA or IL-10R $\alpha$ siRNA, and wound widths were determined 0 and 24 hours after wounding (***P $P<0.001, n=5$, mean \pm SEM). Colonoscopy-based biopsy wounds (2-mm punch biopsies) were generated in C57BL/6 mice and collected on days $1-3$ after injury. Intact tissue was used as a control. These samples were analyzed by qPCR for IL-10 kinetics in intestinal mucosal wounds (D), ELISA (E), and Western blotting (F). (D) I/10 qPCR of intact and wounded tissue on different post-injury days $\left({ }^{*} P<0.05\right.$ and ${ }^{* *} P<0.001 ; n=3$, mean \pm SEM). (E) Punch biopsy samples (2-mm) of resealing colonic wounds on different post-injury days and intact tissue were incubated overnight in complete DMEM. Supernatants were collected, and IL-10 secretion was analyzed by ELISA. ( ${ }^{* *} P<0.001 ; n=3$, mean \pm SEM). (F) Lysates from wounded tissue on different post-injury days and intact tissue were immunoblotted for IL-10 (representative blot is shown, $n=3$ ). Statistical comparisons were performed using ANOVA with Tukey's multiple comparisons post test and a 2-tailed Student's $t$ test. IT, intact tissue; NT, nontransfected; Scr, scramble. ated the expression of IL-10 protein and mRNA in healing biopsyinduced mouse colonic mucosal wounds. Significantly increased Il1O mRNA levels were detected 1 day after injury, with peak levels observed 2 days after injury (Figure 1D; 5 -fold increase, $P<0.001$ ). In parallel studies, IL-10 protein was measured in the supernatants of healing colonic mucosal wounds that were cultured for 4 hours ex vivo. Increased IL-10 protein was detected within 1 day after injury, with continued upregulation on days 2 and 3 (Figure 1E; 3-fold increase compared with non-wounded mucosa on day 2, $P<0.001)$. Figure $1 \mathrm{~F}$ also shows an upregulation of IL-10 protein levels in lysates of mucosal wounds within 1 day after injury. Taken together, these results demonstrate that IL-10 stimulates in vitro intestinal epithelial wound healing and is upregulated during in vivo intestinal mucosal wound repair.

Macrophage-derived IL-10 promotes in vivo intestinal mucosal wound repair. To elucidate the role of IL-10 during in vivo intestinal mucosal wound repair, we compared intestinal mucosal wound healing in IL-10-deficient $\left(I l 10^{-/-}\right)$and WT mice using a colon biopsy-induced injury model $(7,8)$. Since $I l 10^{-/-}$mice can develop age- and microbiota-dependent spontaneous colitis, we used mice that showed no clinical symptoms of colitis (no weight loss, rectal prolapse, or loose stool) and that had low basal levels of the fecal inflammatory marker lipocalin 2 (9) (Supplemental Figure 1 ; supplemental material available online with this article; https:// doi.org/10.1172/JCI90229DS1). As shown in Figure 2A, delayed mucosal wound healing was observed in $\mathrm{Il1O}^{-/-}$mice compared with that in WT mice $\left(21.3 \% \pm 2.53 \%\right.$ in $I l 10^{-/-}$vs. $49.6 \% \pm 2.87 \% \mathrm{WT}$;
$P<0.001) 3$ days after injury. These functional wound-healing data were consistent with histologic analyses confirming increased wound closure. Given that both innate and adaptive immune responses have been reported to be involved in IL-10 secretion and that previous observations have demonstrated that IL-10 production by $\mathrm{CD}^{+} \mathrm{T}$ cells is instrumental in regulating spontaneous colitis (10), we next examined whether adaptive immune cells were required for mucosal wound repair by using RAG1-deficient (Rag1/--) mice that lack mature $\mathrm{T}$ and B cells (11). Notably, mucosal wound repair in $\mathrm{Rag1}^{-/}$mice was indistinguishable from that in WT mice, suggesting that $\mathrm{T}$ and $\mathrm{B}$ cells are not required for mucosal wound repair (Figure 2B; Rag1 1 - $: 47.6 \% \pm 4.21 \%$; WT: $45.5 \%$ $\pm 2.65 \%)$. Since macrophages have also been reported to be an abundant source of IL-10 in the intestinal mucosa, wound healing was next analyzed in mice with a conditional deletion of Il1O in CD11c-expressing cells (Il10 ${ }^{f / f l}$ CD11c-Cre mice, hereafter referred to as IL-10 ${ }^{\mathrm{fl} / \mathrm{fl}} \mathrm{CD} 11 \mathrm{c}$ Cre mice), which are predominantly macrophages and DCs. Analogous to results using global $\mathrm{IllO}^{-/-}$mice, we also observed delayed wound closure in IL-10 $\mathrm{f}^{\mathrm{fl} / \mathrm{fl}}$ CD11c Cre mice when compared with littermate control $\mathrm{IL}-10^{\mathrm{fl} / \mathrm{fl}}$ mice (Figure 2C; IL-10 ${ }^{\mathrm{fl} / \mathrm{fl}} \mathrm{CD} 11 \mathrm{c}$ Cre: $13.84 \% \pm 3.49 \%$; IL-10 ${ }^{\mathrm{fl} / \mathrm{fl}}$ : $41.84 \% \pm 3.78 \% ; P<0.001)$. Analysis of IL-10 protein in intestinal mucosal wounds confirmed increased IL-10 expression in $\mathrm{IL}-10^{\mathrm{f} / \mathrm{fl}}$ mice but not in $\mathrm{IL}-10^{\mathrm{fl} / \mathrm{fl}} \mathrm{CD} 11 \mathrm{c}$ Cre mice (Figure 2D). Furthermore, IL-10 colocalized with the macrophage marker $\mathrm{F} 4 / 80$ in mucosal wound beds in IL-10 GFP reporter mice (Figure $2 \mathrm{E}$ ). To corroborate these morphologic observations, we 
A

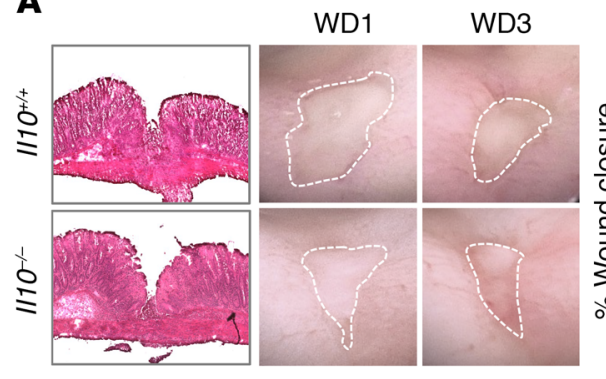

C

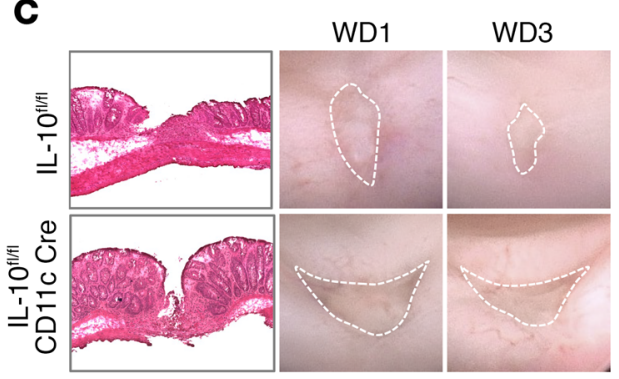

E

IL-10 (GFP)/F4/80/Nuclei/F-actin

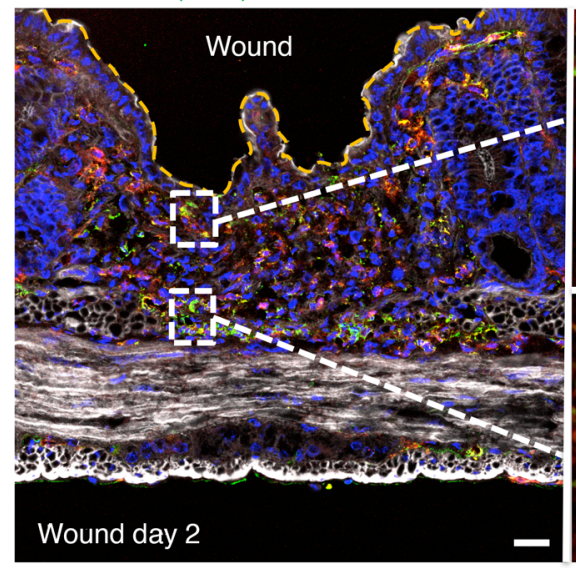

G

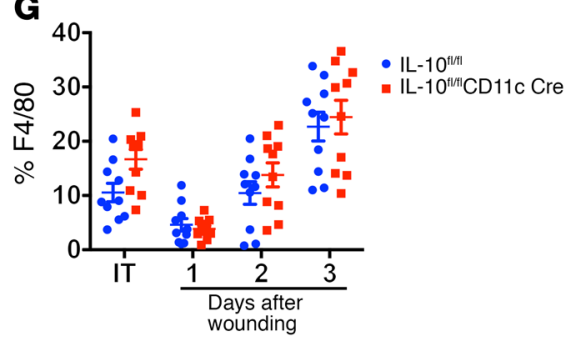

B
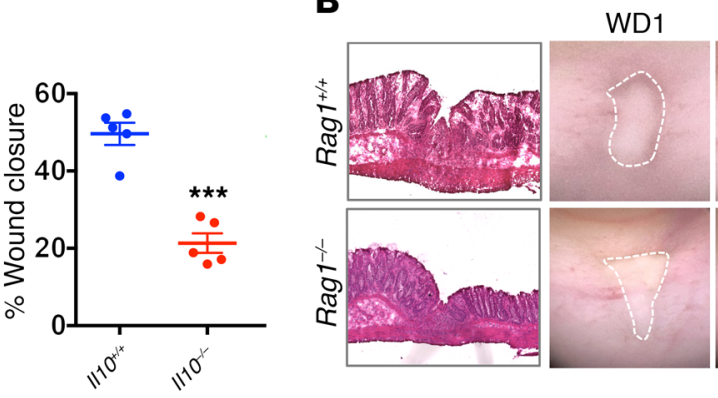

WD3
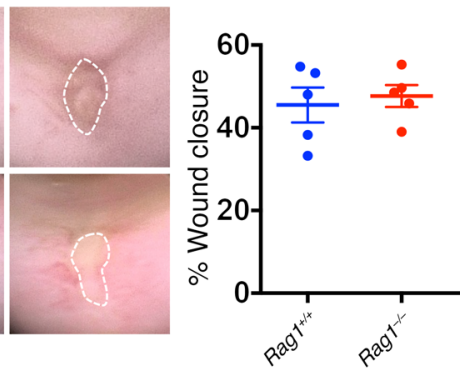

D

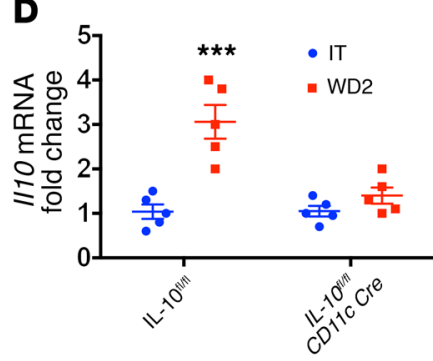

$\mathbf{F}$
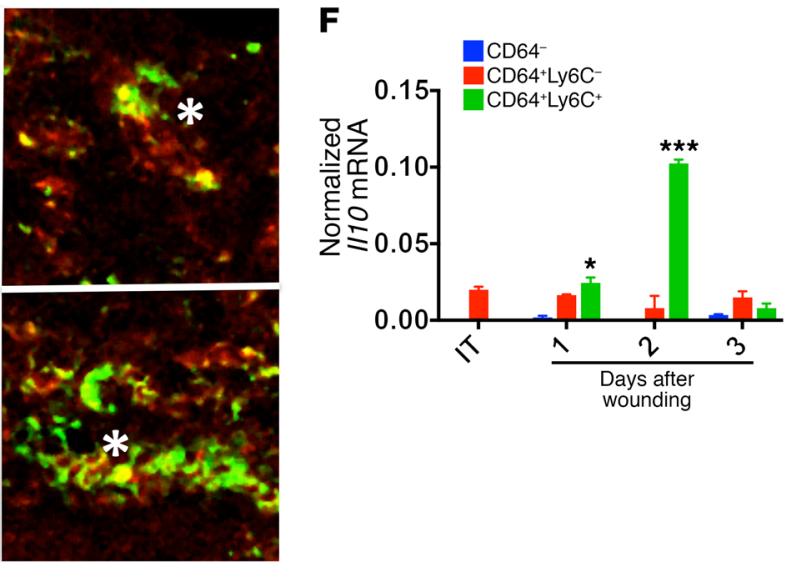

H

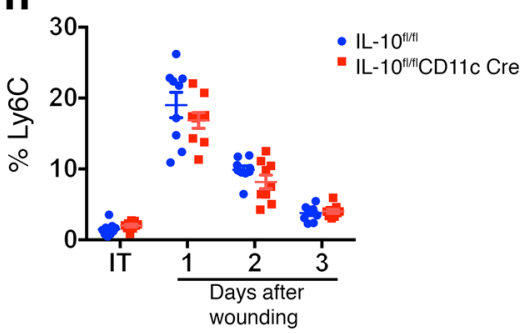

Figure 2. Macrophage-derived IL-10 promotes intestinal mucosal wound healing. (A) Endoscopic images of healing colonic mucosal wounds 1 and 3 days after biopsy-induced injury in $1 / 10^{-/-}$versus $1 / 10^{+/+}$mice and quantification of wound repair (*** $P<0.001 ; n=5$ mice, average of 3 wounds, mean \pm SEM). (B

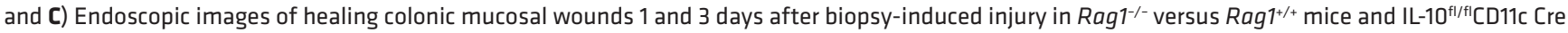
versus IL-10 $10^{f / f 1}$ mice and quantification of wound repair (***P $P<0.001, n=5$ mice, average of 3 wounds, mean \pm SEM). (D) I/10 mRNA levels in intact colonic tissue and day-2 post-injury wounds in IL-10 $\mathrm{fl}^{\mathrm{fl} f \mathrm{C}} \mathrm{CD} 11 \mathrm{c}$ Cre and IL-10 $0^{\mathrm{fl} / \mathrm{fl}}$ mice ${ }^{* * *} P<0.001, n=5$, mean $\pm \mathrm{SEM}$ ). (E) Confocal micrographs of punch biopsies from IL-10 GFP reporter mice with resealing colonic wounds (2 days after injury) showing staining for GFP, F4/80, F-actin (phalloidin), and nuclei. Scale bar: $20 \mu \mathrm{m}$. Original magnification, $\times 40$. White asterisks highlight IL-10-GFP colocalization with the macrophage marker F4-80. (F) I/10 mRNA-normalized levels of immune cells isolated from wounded LP colonic tissue on different post-injury days and intact tissue $\left({ }^{*} P<0.05\right.$ and ${ }^{* * *} P<0.001 ; n=5$ mice, image is representative of 2 separate experiments, mean \pm SEM). ( $\mathbf{G}$ and $\mathbf{H}$ ) Representative plots of LP myeloid cells isolated from intact and wounded tissue

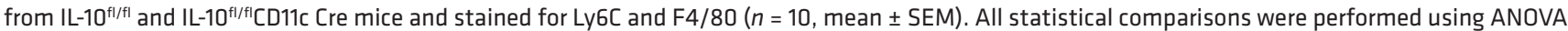
with Tukey's multiple comparisons post test. WD1, 1 day after wounding; WD3, 3 days after wounding. 

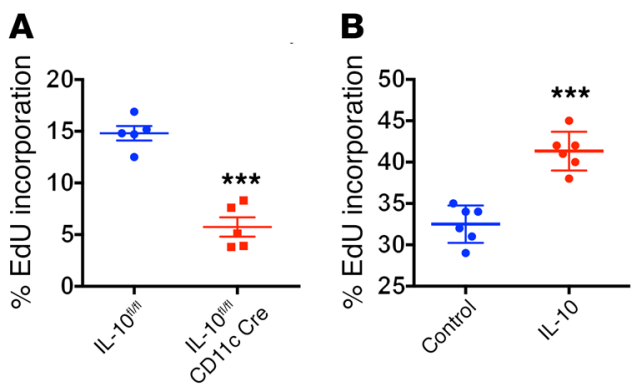

C
D

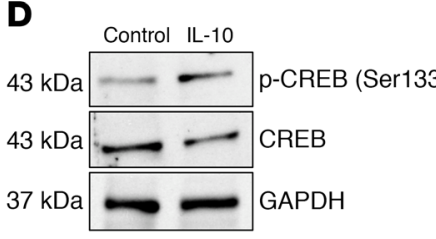

E

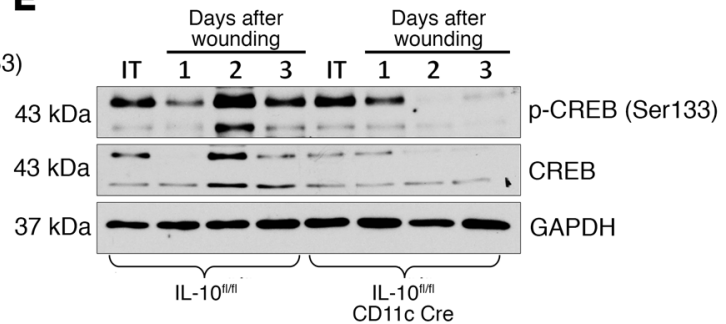

Figure 3. IL-10 promotes IEC proliferation and activates CREB signaling. (A) EdU incorporation was

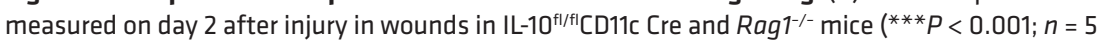
mice, average of 3 wounds, mean \pm SEM). (B) Scratch-wounded intestinal epithelial monolayers were treated with rhIL-10 (100 nM), and EdU incorporation was determined 24 hours after wounding ( ${ }^{* * *} P<0.001 ; n=6$, mean \pm SEM). (C) Immunoblotting for CREB and activated $p$-CREB (Ser133) was performed using cells from scratch-wounded intestinal epithelial monolayers treated with rhIL-10 (100 $\mathrm{nM})$ and Stattic $(10 \mu \mathrm{M})$ for 60 minutes. (D) Primary human enteroid cultures were treated with IL-10 (100 nM) for 24 hours. (E) Lysates from intact colon (IT) and healing wounds harvested on days 1-3 from IL-10 ${ }^{\mathrm{fl} / \mathrm{fl}}$ and IL-10 $0^{\mathrm{fl} / \mathrm{fl}} \mathrm{CD} 11 \mathrm{c}$ Cre mice. C, D, and $\mathbf{E}$ show representative blots from 3 experiments. All statistical comparisons were performed using ANOVA with Tukey's multiple comparisons post test.

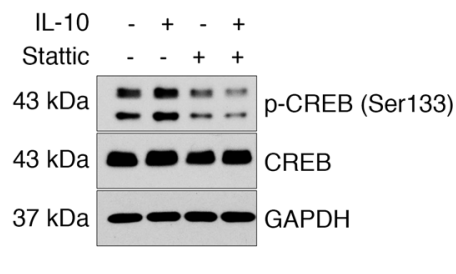

wounding. No significant differences were observed in wound-associated immune cell dynamics for resident macrophages or infiltrating monocytes/macrophages (Figure 2, G-H), as well as DCs (defined as $\mathrm{CD} 45^{+} \mathrm{CD} 103^{+} \mathrm{F} 4 / 80^{-} \mathrm{CD} 64^{-}$), polymorphonuclear neutrophils (PMNs) and PMNs/ myeloid-derived suppressor cells (PMNs/ PMN-MDSCs) (defined as $\mathrm{CD} 45^{+} \mathrm{CD} 11 \mathrm{~b}^{+}$ Ly6 $\mathrm{G}^{+} \mathrm{Ly}_{6 \mathrm{C}^{-}}$), and monocytes/monocyteMDSCs (monocytes/M-MDSCs) (defined as CD $45^{+}$CD $11 b^{+}{ }^{+}$6 6 G ${ }^{-}$Ly6C ${ }^{\text {hi }}$ ) (Supplemental Figure 1, D-G). Taken together, these results support an important role for infiltrating monocyte/macrophage-derived IL-10 in promoting intestinal mucosal wound repair.

IL-10 promotes intestinal epithelial cell proliferation by CREB signaling at the sites of mucosal injury. Coordinated epithelial cell proliferation and migration mediate wound closure and restore mucosal homeostasis. To identify the mechanism by which IL-10 promotes wound repair, we analyzed the effect of IL-10 on epithelial cell proliferation during mucosal wound repair. We analyzed the incorporation of a thymidine analog, $\mathrm{EdU}$, in the intestinal epithelium in healing intestinal wounds from IL-10 ${ }^{\mathrm{f} / \mathrm{fl}}$ and $\mathrm{IL}-10^{\mathrm{f} / \mathrm{fl}}$ CD11c Cre mice. As shown in Figure 3A, quantified Il1O mRNA in immune cells isolated from the lamina propria (LP) of either intact or wounded WT mouse colon on days 1-3 after injury. We sorted immune cells into 3 populations: non-macrophages $\left(\mathrm{CD} 45^{+} \mathrm{CD} 64^{-} \mathrm{Ly} 6 \mathrm{C}^{-}\right)$; resident macrophages $\left(\mathrm{CD}_{4} 5^{+} \mathrm{CD} 64^{+} \mathrm{Ly}^{-} \mathrm{C}^{-}\right)$; and infiltrating macrophages $\left(\mathrm{CD} 45^{+} \mathrm{CD} 64^{+} \mathrm{Ly} 6 \mathrm{C}^{+}\right)$. Our results show that, among these 3 populations, infiltrating macrophages $\left(\mathrm{CD} 45^{+} \mathrm{CD} 64^{+} \mathrm{Ly} 6 \mathrm{C}^{+}\right)$had the highest $I l 10$ mRNA expression levels on day 2 after injury, suggesting that macrophages are a main source of IL-10 in the wound bed (Figure 2F). To evaluate the role of the microbiota in the increase of IL-10 after mucosal injury, we compared Il1O mRNA levels in intact and day-2 post-wound tissue isolated from specific pathogenfree (SPF) or germ-free (GF) mice. As shown in Supplemental Figure 1C, GF mice had a reduction in steady-state levels of Il1O mRNA when compared with levels in SPF mice. Following mucosal injury, however, Il1O mRNA was induced in post-wound colonic tissue isolated from both SPF and GF mice, suggesting that the injury-triggered increase in IL-10 is largely microbiota independent. We next examined the phenotypic transition of infiltrating immune cell populations in the wound bed of $I L-10^{\mathrm{f} / \mathrm{fl}}$ and $\mathrm{IL}-10^{\mathrm{f} / \mathrm{fl}} \mathrm{CD} 11 \mathrm{c}$ Cre mice on post-injury days $1-3$ and in intact tissue. As shown in Figure 2G, nearly all macrophages in intact colonic tissue had a resident phenotype $\left(\mathrm{CD} 45^{+} \mathrm{CD} 64^{+} \mathrm{Ly} 6 \mathrm{C}^{-}\right)$, regardless of mouse genotype. On day 1 after wounding, we observed a strong accumulation of infiltrating monocytes/ macrophages $\left(\mathrm{CD} 45^{+} \mathrm{CD} 64^{+} \mathrm{Ly} 6 \mathrm{C}^{\mathrm{hi}} \mathrm{F} 4 / 80^{\mathrm{lo}}\right.$ ) (Figure $2 \mathrm{H}$ ), and this cell population appeared to transition into a resident macrophage phenotype $\left(\mathrm{CD} 45^{+} \mathrm{CD} 64^{-} \mathrm{Ly} 6 \mathrm{C}^{\mathrm{lo}} \mathrm{F} 4 / 80^{\mathrm{hi}}\right)$ as early as day 2 after decreased intestinal epithelial cell proliferation was observed in $\mathrm{IL}-10^{\mathrm{f} / \mathrm{fl}} \mathrm{CD} 11 \mathrm{c}$ Cre mice compared with that seen in $\mathrm{IL}-10^{\mathrm{f} / \mathrm{fl}}$ mice $\left(14.81 \% \pm 0.70 \% \mathrm{IL}-10^{\mathrm{fl} / \mathrm{fl}}\right.$ vs. $5.74 \% \pm 0.93 \% \mathrm{IL}-10^{\mathrm{f} / \mathrm{f} \mathrm{C}} \mathrm{CD} 11 \mathrm{c}$ Cre $P<$ 0.001). To corroborate these findings, we measured epithelial cell proliferation in SKCO-15 intestinal epithelial cells (IECs) treated with recombinant human IL-10 (rhIL-10). As shown in Figure 3B, treatment with rhIL-10 increased IEC proliferation $(32.5 \% \pm 0.92 \%$ control vs. $41.33 \% \pm 0.95 \%$ IL-10; $P<0.001)$. Since IL-10 promotes epithelial cell proliferation and wound repair, we next investigated the underlying mechanisms of this response. As cAMP response element-binding protein (CREB) signaling has been linked to cell proliferation, we determined whether IL-10 activates CREB in a STAT3-dependent manner by inducing its phosphorylation at serine 133. As shown in Figure 3, C and D, IL-10 increased CREB phosphorylation in SKCO-15 cells as well as in primary human epithelial cell cultures (enteroids) (densitometric analysis, Supplemental Figure 2, A and B). Additionally, we detected increased phosphorylation of CREB (Ser133) in in vivo biopsy-induced wounds 3 days after injury in $\mathrm{IL}-10^{\mathrm{f} / \mathrm{fl}}$ mice (Figure 3E, densitometric results in Supplemental Figure 2C). We did not observe this increase in CREB phosphorylation in $\mathrm{IL}-1 \mathrm{O}^{\mathrm{f} / \mathrm{f}} \mathrm{CD} 11 \mathrm{c}$ Cre mice. We also observed that CREB inhibition did not influence the induction of classic IL-10 downstream target proteins such as c-myc (Supplemental Figure 2D). Taken together, these results suggest that IL-10 activates CREB signaling.

$I L-10$ activation of CREB signaling promotes WISP-1 secretion. Since IL-10 induces CREB activation in human IECs, we next explored the mechanisms by which IL-10-mediated activation of CREB signaling promotes epithelial cell proliferation and, ulti- 
A

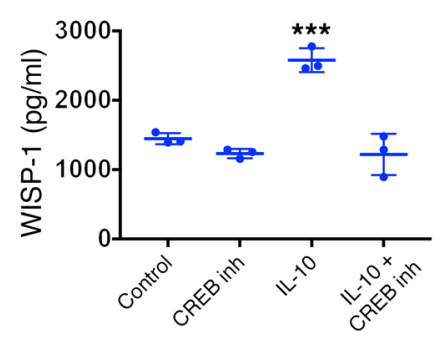

B

-WISP1 promoter

- $\triangle$ CREB WISP1 promoter
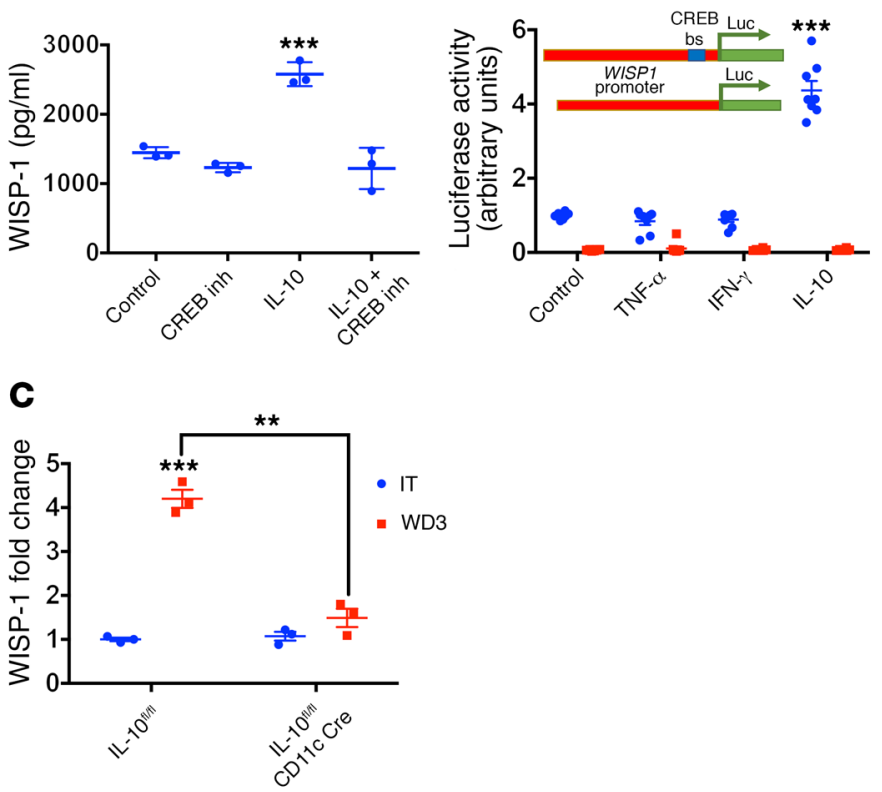

Figure 4. IL-10 promotes WISP-1 secretion in a CREB-dependent fashion. (A) WISP-1 levels were measured in supernatants from grid-wounded intestinal epithelial monolayers with and without incubation with hrlL-10 (100 $\mathrm{nM}$ ) and/or the CREB inhibitor 92-78-4 (inh) for 24 hours ( ${ }^{* *} P<0.001, n=3$, mean \pm SEM). (B) SKCO-15 cells transfected with a WISP1 promoter coupled to luciferase (Luc) with or without the CREB-binding site were treated with TNF- $\alpha$, IFN- $\gamma$, IL-10, or BSA $(100 \mathrm{nM})\left({ }^{* * *} P<0.001, n=8\right.$, mean \pm SEM). bs, binding site. (C) Lysates from intact colon and wounds harvested on day 3 from IL-10 ${ }^{f / / f \mid} C D 11 c$ Cre and IL-10 ${ }^{f / / f l}$ mice $\left(^{* * *} P<0.001\right.$ and ${ }^{* *} P<0.01, n=3$, mean \pm SEM). All statistical comparisons were performed using ANOVA with Tukey's multiple comparisons post test. inh, inhibitor.

mately, wound healing. WISP-1 is a CREB target protein that has been reported to promote epithelial cell proliferation (12). Since WISP-1 is a secreted protein, we examined the influence of IL-10 on WISP-1 secretion from healing IEC wounds. As shown in Figure $4 \mathrm{~A}$, rhIL-10 treatment of IEC induced WISP-1 secretion, and this response was inhibited by coincubation with a pharmacological CREB-CBP inhibitor (CREB-binding protein) $(P<0.001)$ or by knocking down IL-10R $\alpha$ expression (Supplemental Figure 3A). It has been previously reported that the WISP1 gene promoter contains a CREB-binding site that mediates its transcriptional activation (12). Thus, to examine the relationship between IL-10 induction and WISP-1 secretion, we examined the influence of IL-10 on WISP1 promoter activity. Increased WISP1 luciferase reporter activity was observed in IECs incubated with IL-10 (Figure $4 \mathrm{~B} ; 4.36- \pm 0.253$-fold), and this effect was abrogated in cells transfected with a WISP1 luciferase reporter construct containing mutations in the CREB-binding site. A positive control for this experiment using forskolin to activate CREB signaling showed a 8.21-fold increase in luciferase activity (Supplemental Figure 3B). Since treatment with exogenous IL-10 increased WISP-1 secretion in healing wounds in vitro, we next examined whether WISP-1 production in vivo was influenced by macrophage-derived IL-10. Intestinal mucosal levels of WISP-1 in punch biopsies 3 days after wounding were measured in IL-10 $0^{\mathrm{f} / \mathrm{l}}$ and $\mathrm{IL}-10^{\mathrm{f} / \mathrm{fl}} \mathrm{CD} 11 \mathrm{c}$ Cre mice
(Figure 4C). We found that the increased WISP-1 secretion in healing wounds from IL- $10^{\mathrm{f} / \mathrm{l}}$ mice was absent in $\mathrm{IL}-10^{\mathrm{f} / \mathrm{ll}} \mathrm{CD} 11 \mathrm{c}$ Cre mice (4.2- \pm 0.20 -fold increase in IL-10 $0^{\mathrm{f} / \mathrm{l}}$ mice vs. 1.49- \pm 0.21 fold increase in IL-10 ${ }^{\mathrm{A} / \mathrm{l}} \mathrm{CD} 11 \mathrm{c}$ Cre mice; $P<0.01$ ). In contrast, the WISP-1 secretion from intact healthy intestinal mucosa was comparable between these groups of mice.

WISP-1 is secreted in response to intestinal mucosal injury. To corroborate our in vitro findings, we analyzed WISP1 mRNA levels in healing biopsy-induced intestinal mucosal wounds. As shown in Figure 5A, increased WISP1 mRNA was identified in healing intestinal mucosal wounds 3 days after injury (day $3, P<0.001$ ). Immunofluorescence labeling identified WISP-1 protein in crypt base intestinal epithelial cells and in LP cells, with increased WISP-1 expression detected in the repairing epithelium adjoining the mucosal injury (Figure 5B and Supplemental Figure 4). To support these data, we determined WISP1 mRNA levels in human colon samples from both healthy and active IBD tissue. As shown in Figure 5C, WISP1 mRNA levels were increased in colonic tissue with an active inflammatory process. Immunofluorescence labeling showed that WISP-1 was expressed in the base of healthy human colonic crypts, with a prominent increase in expression detected in colonic crypts from active colitis tissue (Figure 5D). Furthermore, Western blot analysis of human colonic mucosal samples from healthy individuals and individuals with chronic active colitis revealed increased levels of WISP-1 protein in IBD samples compared with levels in healthy control samples. Similarly, IL-10R $\alpha$ expression was increased (Figure 5E). Immunoblots for the IFN- $\gamma$-induced gene GTPase guanylate-binding protein 1 (GBP1) in IBD samples revealed increased protein levels, supporting the finding of increased active inflammation in tissue samples (13). Taken together, these results suggest that WISP-1, IL-10R $\alpha$, and GBP- 1 are upregulated in the intestinal mucosa in response to injury and inflammation.

WISP-1 promotes intestinal epithelial wound repair. Since IL-10 promotes WISP-1 synthesis and secretion, we determined whether WISP-1 enhances intestinal epithelial wound repair using the model intestinal epithelial cell lines SKCO-15 and IEC-6. As shown in Figure 6, recombinant human WISP-1 (rhWISP-1) increased epithelial wound closure in a time- and dose-dependent manner (Figure 6A, $P<0.001$, and Supplemental Figure 5, A and B). Furthermore, the reparative effects of WISP-1 were inhibited with WISP-1-inhibitory Abs (Figure 6A, $P<0.05$ ). Additionally, siRNA-mediated knockdown of WISP-1 (Supplemental Figure 5C) inhibited the increased wound closure following treatment with IL-10 and WISP-1 (Figure 6B and Supplemental Figure 5D). To determine whether epithelial cell proliferation contributes to the increased wound closure mediated by WISP-1, we analyzed EdU incorporation of healing epithelial wounds in vitro. Increased intestinal epithelial cell proliferation in response to WISP-1 incubation was inhibited with a WISP-1-inhibitory Ab (Figure 6C) or by siRNA-mediated knockdown WISP-1 expression (Figure 6D). To determine whether WISP1 induces gene expression programs that promote intestinal epithelial wound healing, we performed a transcription factor PCR array. Figure 6E shows WISP-1-induced upregulation of POU5F1 (OCT4) and $N A N O G$, both key pluripotency genes implicated in proliferation and cell renewal. We also found that IECs treated for 24 hours with rhWISP-1 have increased transactivation of TCF/LEF reporter 
A

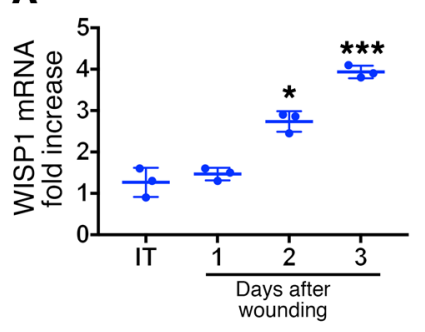

B

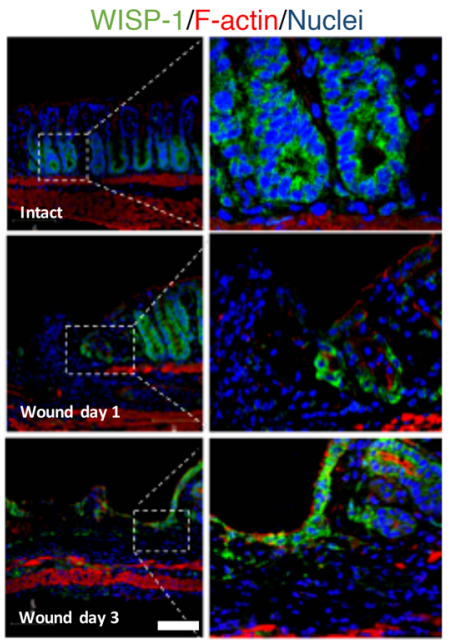

C

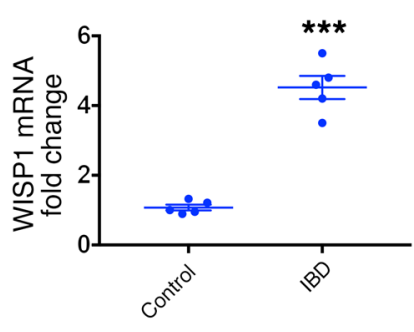

D

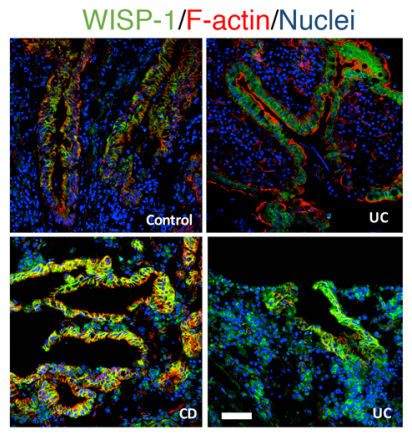

E

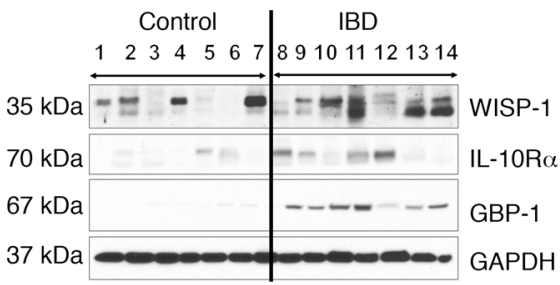

Figure 5. Colonic injury upregulates WISP-1 synthesis and secretion. (A) WISP1 mRNA levels were determined in tissue obtained from punch biopsies of intact colon (IT) and wounded colonic mucosa on post-injury days 1,2 , and 3 ( ${ }^{* *} P<0.001$ and ${ }^{*} P<0.05, n=3$, mean \pm SEM). (B) Laser confocal micrographs of frozen sections from intact colon and wounds on days 1 and 3 after wounding show WISP-1 expression (green), F-actin (red), and nuclei (blue). Scale bar: $100 \mu \mathrm{m}$. Original magnification $\times 20$. (C) WISP1 mRNA levels in colon biopsies from healthy controls and IBD patients with active inflammation in the colon ( ${ }^{* *} P<0.001, n=5$, mean \pm SEM). (D) Laser confocal micrographs of frozen sections from healthy and active IBD tissue show WISP-1 expression (green), F-actin (red), and nuclei (blue). Scale bar: $100 \mu \mathrm{m}$. UC, ulcerative colitis; CD, Crohn's disease. (E) Lysates of healthy and active IBD tissue from human colon samples were immunoblotted to detect WISP-1, IL-10R $\alpha$, GBP-1, and GAPDH. B, D, and E show representative images from 3 experiments. Sample information pertaining to patient diagnosis, age, and treatment is provided in Supplemental Table 1. All statistical comparisons were performed using ANOVA with Tukey's multiple comparisons post test.

activity, which was analyzed using the TOP/FOP luciferase reporter assay (Supplemental Figure 5E). These findings support a role for WISP-1 in activating the WNT signaling pathway. Previous studies have shown that c-myc, a pro-proliferative protein, is a direct target gene of the WNT signaling pathway in the intestine (14). As shown in Figure 6F (densitometry in Supplemental Figure 5F), IL-10 and WISP-1 incubation increased intestinal epithelial cell c-myc protein levels. Last, we analyzed the functional effects of WISP-1 on wound repair in vivo by administration of WISP-1-inhibitory Ab (15) in the colonic mucosa of healing biopsy-induced wounds. The administration of WISP-1-inhibitory Ab into healing intestinal mucosal wounds impaired wound repair relative to isotype control $\mathrm{Ab}$ administration $(45.6 \% \pm 2.99 \% \operatorname{IgG}$ isotype vs. $13.2 \% \pm 2.41 \%$ WISP-1-blocking Ab, $P<0.001$ ) (Figure 6G). Taken together, these findings support an important role for WISP-1 in promoting intestinal mucosal wound repair.

\section{Discussion}

Epithelial damage associated with disruption of the intestinal mucosal barrier occurs following mechanical injury and is pathognomonic of a number of diseases including IBD (16). Repair of intestinal injury and the regaining of mucosal homeostasis require an actively induced and coordinated response that culminates in the migration and proliferation of IECs to cover denuded mucosal surfaces and reestablish the mucosal barrier. This re-epithelialization is facilitated by complex interactions between mediators derived from the epithelium as well as from resident and infiltrating immune cells
(2). One of the mediators previously implicated in epithelial repair is the antiinflammatory cytokine IL-10, which plays a protective role during intestinal inflammation and is critical for the maintenance of intestinal homeostasis (3). As such, $I l 10^{-/}$mice have an amplified, microbiota-dependent immune response that leads to overproduction of proinflammatory cytokines and culminates in spontaneous enterocolitis (4). While the role of IL-10 signaling on immune cell function has been well characterized, the effects of this cytokine on repair of the mucosal barrier and the cellular sources of IL-10 secretion following intestinal mucosal injury have not yet been fully elucidated. Previous studies have reported an important role of microbiota in mucosal IL-10 generation under homeostatic conditions (17-19). GF mice have a 50\% reduction in IL-10 steady-state levels. Here, we report that IL-10 is synthesized and secreted in response to colonic mucosal injury in vivo, and this response is not dependent on microbiota exposure. Furthermore, the peak expression of IL-10 is observed in a transition between the pro-inflammatory and restorative phases of wound closure.

We further identified the importance of IL-10 in promoting mucosal wound repair by utilizing $I l 10^{-/-}$mice that exhibit delayed colonic mucosal wound healing when compared with WT mice. Earlier reports have identified increased IL-10 levels following injury of rat sciatic nerve, and a beneficial role of IL-10 has been implicated in tissues such as cardiac muscle, carotid endothelia, and lung epithelia (20-22). We found that epithelial IL-10R $\alpha$ mediates the IL-10-driven increase in wound healing. Previous studies have addressed the importance of IL-10R $\alpha$ 
A

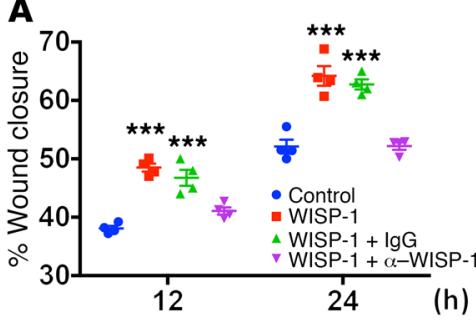

\section{(h)}
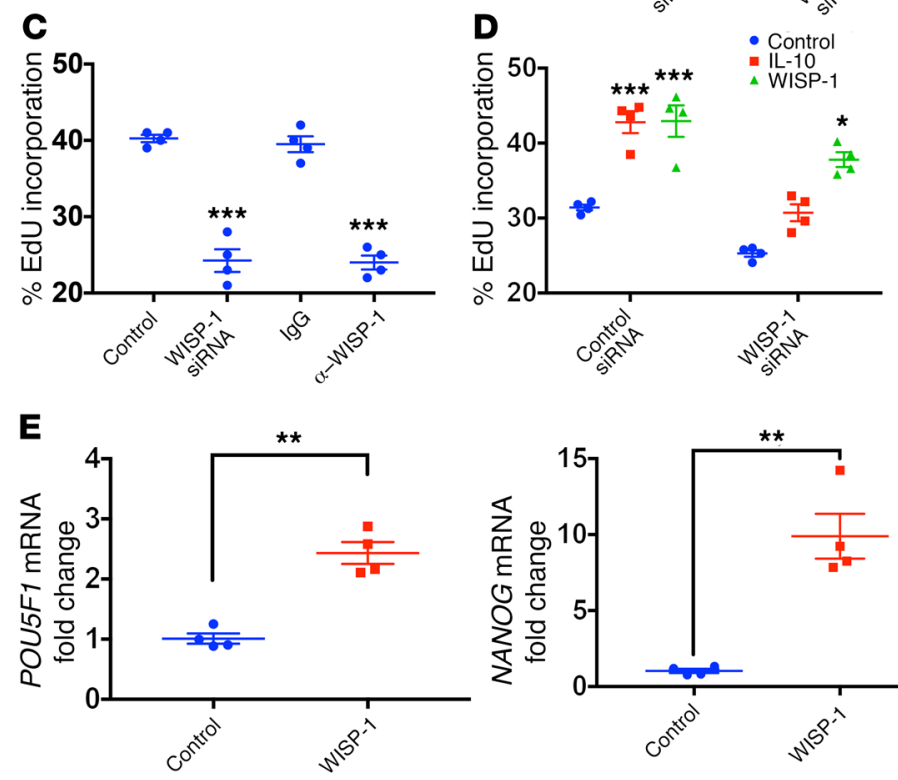

\section{$\mathbf{F}$}

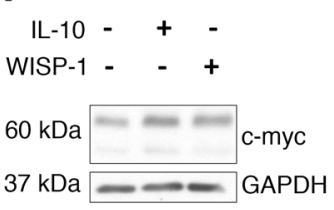

G
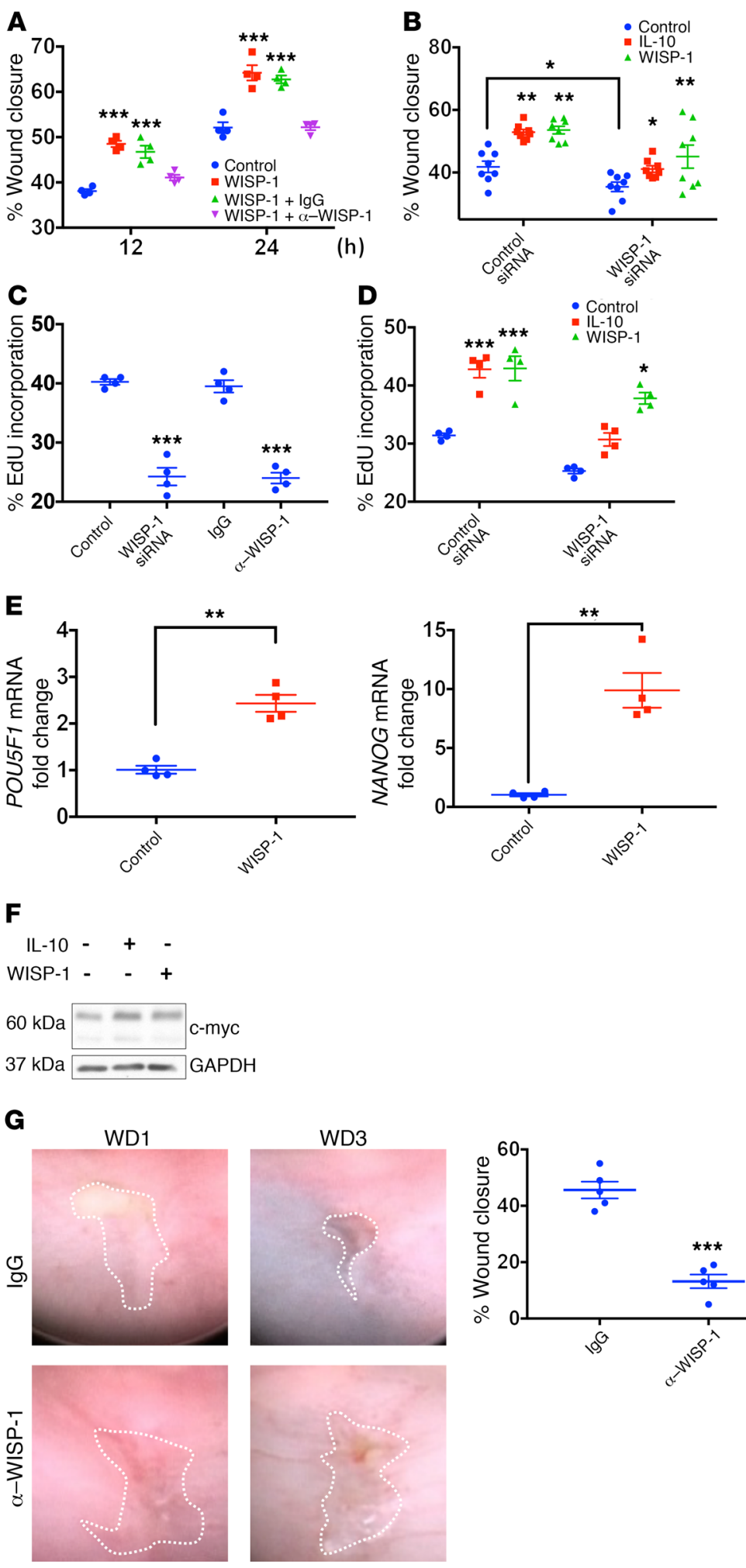

Figure 6. WISP-1 promotes intestinal epithelial wound healing. (A) Intestinal epithelial wound closure percentage was calculated by measuring wound widths 0,12 , and 24 hours after wounding $\left({ }^{* *} P<0.001, n=4\right.$, mean $\left.\pm S E M\right)$. Cells were incubated with WISP-1 $(500 \mathrm{nM})$ in the presence of WISP-1-inhibitory Abs ( $\alpha$-WISP-1) $(10 \mu \mathrm{g} /$ wound) and IgG-matched control Abs. (B) Percentage of wound closure in cells after siRNA-mediated downregulation of WISP-1 (WISP-1 siRNA) or scramble control siRNA $\left({ }^{*} P<0.05\right.$ and ${ }^{* *} P<0.01 ; n=8$, mean \pm SEM) and treatment with rhIL-10 (100 nM), WISP-1 (500 nM), or BSA. (C and D) Epithelial cell proliferation was determined by analysis of EdU incorporation in control epithelial cells, cells with siRNA-mediated downregulation of WISP-1 (WISP-1 siRNA), WISP-1-inhibitory Abs with corresponding IgG-matched control ( $10 \mu \mathrm{g} /$ well). (C, ${ }^{* *} P<0.001, n=4$, mean $\pm \mathrm{SEM}$ ), and in cells treated with rhIL-10 (100 nM), WISP-1 (500 nM), or BSA (D, ${ }^{*} P<0.05$ and ${ }^{* *} P<0.001, n=4$, mean \pm SEM). (E) POU5F1 and NANOG qPCR of SKCO-15 cells treated with WISP-1 (500 nM) or BSA ( ${ }^{* *} P<0.01 ; n=3$, mean $\left.\pm S E M\right)$. (F) Intestinal epithelial cells from scratch-wounded monolayers were treated with BSA, IL-10 (100 nM), or WISP-1 (500 nM) for 2 hours. Harvested cells were immunoblotted for the pro-proliferative protein c-myc and the loading control GAPDH. Blot is representative of 3 experiments. (C) Endoscopic images of healing mucosal wounds 1 and 3 days after biopsy-induced injury in WT mice that were administered WISP-1-neutralizing Ab or an IgC isotype control into the wound bed $\left({ }^{* *} P<0.001, n=5\right.$, mean \pm SEM). All statistical comparisons were performed using ANOVA with Tukey's multiple comparisons post test and a 2-tailed Student's $t$ test.

the wound and by increasing pro-resolution responses including regulation of the extracellular matrix and optimization of fibroblast function $(24,25)$. While it has been previously shown that IECs can produce IL-10 at sites of mucosal injury $(26,27)$, infiltrating immune cells are an abundant source of this cytokine $(5,11,28,29)$. Therefore, it is likely that the source of IL-10 produced during inflammation and subsequent repair of intestinal tissues changes in a temporal fashion depending on the composition of the immune cell compartment. Given previous reports of IL-10 generation by epithelial cells and neutrophils, it is possible that, in our model, these cells contributed to increased IL-10 generation within 1 day after injury. Nevertheless, the highest levels of IL-10 were detected in day-2 healing mucosal wounds. This time point corresponds with a robust macrophage infiltration of the repairing mucosa, suggesting that macrophages could represent an important source of IL-10 in healing mucosal wounds. In support of this, it has been previously reported that IL-10 is robustly expressed by intestinal LP macrophages following mucosal barrier compromise and exposure to luminal microbes (17).

in the colon (23). It has been shown that intestinal epithelial IL-10R $\alpha$ is apically expressed, is upregulated during inflammation, and promotes barrier recovery.

Once secreted at sites of mucosal injury, IL-10 exerts both antiinflammatory and pro-resolution effects. This is mediated through limiting the immune inflammatory response at the site of
Macrophages play a critical role in mucosal wound healing because of their well-characterized role in the generation of factors that stimulate both angiogenesis and fibroplasia $(30,31)$. For example, skin wounds in mice depleted of macrophages have delayed wound closure due to decreased angiogenesis and secretion of growth factors such as TGF- $\beta$ (32). Furthermore, macro- 


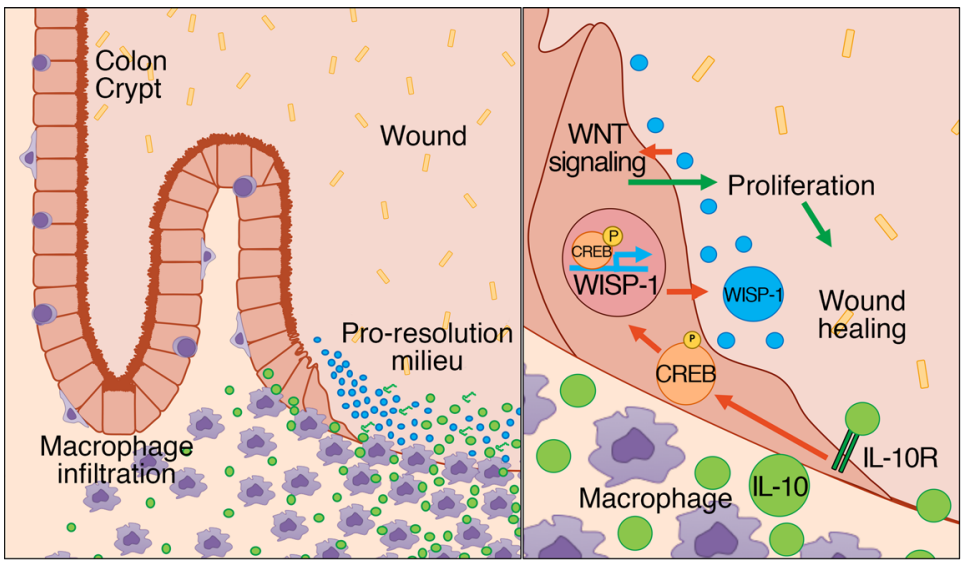

Figure 7. Schematic showing macrophage-derived IL-10 activation of CREB signaling in epithelial cells, leading to WISP-1 generation and wound repair. Macrophages recruited to sites of colonic mucosal injury secrete IL-10, leading to activation of CREB signaling, which increases epithelial WISP-1 secretion that in turn promotes $\beta$-catenin/TCF signaling, epithelial cell proliferation, and wound closure in the intestine.

phage depletion during the early proliferative stage of skin wound closure results in hemorrhage and delayed wound healing (33). In support of the idea that macrophages are a significant source of IL-10 in healing intestinal mucosa, we report here that mice lacking IL-10 expression in CD11c-expressing cells had a deficit in intestinal wound repair, while mice lacking mature $\mathrm{T}$ and $\mathrm{B}$ cells $\left(\mathrm{Rag}^{\wedge}\right.$ - mice) had normal levels of intestinal wound repair. This response can most likely be attributed to the absence of macrophage-derived IL-10, since intestinal DCs are not known to express high levels of IL-10 $(5,10)$. In addition, immunostaining data revealed IL-10 protein in F4/80-expressing macrophages in healing colonic mucosal wounds $(5,10)$. This finding was further supported by digital droplet PCR analysis of sorted macrophage populations in healing colonic mucosal wounds $(5,10)$. The highest expression of $\mathrm{Il1O}$ mRNA was detected in $\mathrm{CD} 45^{+} \mathrm{CD} 64^{+} \mathrm{Ly} 6 \mathrm{C}^{+}$ immune cells, which most likely represented infiltrating monocytes (34). Collectively, our results provide insight into how IL-10producing inflammatory monocytes rapidly accumulate in the intestinal would bed to promote repair processes as they transition to a resident macrophage phenotype.

Analysis of mechanisms by which IL-10 promotes mucosal wound healing identified its influence on intestinal epithelial cell proliferation. Previous studies have reported that IL-10 induced the proliferation of other cell types including B cells, mast cells, and lung fibroblasts (35-37). In addition, accelerated wound healing in the skin of $I l 10^{-/-}$mice (38) has been attributed to other mechanisms that include IL-10 effects on mechanical strength and epithelial organization during repair. Here, we show for the first time to our knowledge that mucosal macrophage-derived IL-10 activates intestinal epithelial CREB protein to promote proliferation and repair, supporting the idea that these molecules play an important role in mucosal repair. CREB is a ubiquitously expressed nuclear transcription factor that is phosphorylated (Ser133) and activated by extracellular stimuli (39). Importantly, CREB is known to regulate genes important for cell proliferation, differentiation, adaptation, and survival in many cell types including
$\mathrm{T}$ and B lymphocytes, hepatocytes, and neural stem cells (40-42). Furthermore, CREB signaling controls the development and homeostasis of epithelial tissues such as those in the lungs and ovaries (43-45). Our study demonstrates a STAT3-dependent link between IL-10 and epithelial CREB signaling that serves to promote epithelial cell proliferation and repair. IT has been previously reported that CREB activation promotes the expression of a pro-proliferative WISP-1 protein that belongs to the CCN family of extracellular matrix-associated proteins $(6,12)$. Our study supports the idea that IL-10-induced epithelial WISP-1 secretion in epithelial cells is mediated by CREB activation.

Chronic inflammatory processes can be associated with an impaired resolution response due to the overwhelming persistence of the proinflammatory milieu that tips the balance toward injury rather than repair (46). We found increased levels of IL-10 and WISP-1 in healing colonic wounds. Increased WISP-1 levels found in inflamed colonic tissue from patients with active colitis suggest that this protein is secreted upon epithelial injury. Although we cannot completely exclude an influence of immunosuppressant therapy on WISP-1 expression, we hypothesize that active inflammation drives WISP-1 expression in human IBD. IL-10 levels are also high in patients with active ulcerative colitis (47), suggesting that these mechanisms come into play during a mucosal inflammatory response that serves to help with the repair process. Increased WISP-1 levels have been identified in repairing skin wounds, and WISP-1-KO mice have delayed skin wound healing (48). Additionally, IL-10 has also been reported to influence keratinocyte wound closure (25).

We observed that WISP-1 upregulates IEC mRNA levels of POU5F1 (encoding OCT4) and NANOG, both of which are key pluripotency genes implicated in proliferation and cell renewal (49). POU5F1 is a WNT target gene that promotes the expression of NANOG (50). These findings are consistent with our results showing increased TCF/LEF transactivation in response to WISP-1 signaling (Supplemental Figure 5E). We propose that WISP-1 acts as a positive feedback loop for the WNT signaling pathway, resulting in upregulation of the pro-proliferative-and pluripotency-related proteins c-myc, OCT4, and NANOG. The increased expression of these genes is consistent with the functional increase in epithelial cell proliferation and wound healing in response to WISP-1. A previous study has reported an influence of WISP-1 in promoting the proliferation of primary human lung fibroblasts that is mediated by IL-6 signaling (51). Taken together, these data highlight an important macrophage-epithelium crosstalk that is mediated by IL-10, CREB, and WISP-1 and that serves to promote healing of mucosal wounds (Figure 7).

\section{Methods}

Mice. C57BL/6 (WT), Rag1 ${ }^{-/}$, Il10 ${ }^{-/}$, and CD11c-Cre (CD11c Cre) mice were purchased from The Jackson Laboratory. CD11c Cre mice were bred with $\mathrm{IL}-10^{\mathrm{t} / \mathrm{ll}}$ mice (52). GF mice were obtained from the University of Michigan GF facility, and all mice were maintained under SPF conditions.

Cell lines and culture conditions. IECs (SK-CO15 [human] and IEC-6 [rat]) were obtained from Enrique Rodriguez-Boulan (Cornell University, Ithaca, New York, USA) and the ATCC, respectively. Human colonic 
enteroids were provided by Jason Spence (University of Michigan, Ann Arbor, MI, USA). IECs were grown in high glucose (4.5 g/l), DMEM supplemented with $10 \%$ FBS, 100 units $/ \mathrm{ml}$ penicillin, $100 \mu \mathrm{g} / \mathrm{ml}$ streptomycin, $15 \mathrm{~mm}$ HEPES (pH 7.4), 2 mm L-glutamine, and 1\% nonessential amino acids, as described previously $(7)$, at $37^{\circ} \mathrm{C}$ in a $4 \% \mathrm{CO}_{2}$ incubator.

IEC monolayer wounding in vitro. Cell migration was assessed using a scratch wound assay as previously published (7). Inhibitors were added 30 minutes prior to mechanical wounding. For immunoblot analysis, confluent cell monolayers grown in 6-well tissue culture plates and grid wounds were generated to enrich for migrating and spreading cells.

In vivo wounding of colonic mucosa. Mice were anesthetized by i.p. injection of a ketamine solution $(100 \mathrm{mg} / \mathrm{kg}) / x y l a z i n e ~(10 \mathrm{mg} / \mathrm{kg})$. A high-resolution, miniaturized colonoscope system equipped with biopsy forceps (Colorview Veterinary Endoscope; Karl Stortz) was used to biopsy-injure the colonic mucosa at 3 to 5 sites along the dorsal artery, and healing was quantified on post-injury days 1 and 3 . Endoscopic procedures were viewed with high-resolution $(1,024 \times 768$ pixels $)$ images on a flat-panel color monitor. Wound sizes averaged approximately $1 \mathrm{~mm}^{2}$, which is equivalent to the removal of approximately 250 to 300 crypts. GF animals were maintained under sterile conditions. For each analysis, 10-15 lesions from 5 mice per group were examined. Mucosal wounds and intact mucosa were harvested for analysis of mRNA expression (realtime PCR), immunoblotting for changes in expression or activation of proteins involved in epithelial cell proliferation and wound closure, as well as immunofluorescence labeling.

Cell proliferation. IEC monolayers were incubated with the different treatments for 24 hours. One hour before harvesting of cells, EdU was added to the media at a final concentration of $10 \mu \mathrm{M}$. For in vivo studies, EdU was injected i.p. (160 $\mu \mathrm{g} / \mathrm{g}$ BW), and after 2 hours, the mice were euthanized and the colon was harvested, rolled, and embedded in OCT for cryosectioning. Proliferating cells were detected with the Click-iT EdU Alexa Fluor 488 Imaging Kit (Life Sciences, Thermo Fisher Scientific; catalog C10637) using a Leica SP5 confocal microscope (Leica Microsystems).

siRNAs. WISP-1 siRNAs (no. 1: GGAGUUUGCAUGGACAAUAGGUGCT; no. 2: ACAUCCAUACACUCAUUAAGGCAGG; no. 3: AGACUAUCGACGUGUCCUUCCAGTG) were purchased from OriGene (catalog SR305836), and IL-10R $\alpha$ and IL-10 $\beta$ siRNAs (SMARTpool, catalog L-007925-00-0005) were purchased from GE Dharmacon and used for transfection studies with Lipofectamine 2000 (Life Technologies, Thermo Fisher Scientific) according to the manufacturers' instructions. Transfection of nonsilencing siRNA (Sigma-Aldrich; catalog SIC002) was used as a negative control.

Immunoblotting and immunofluorescence. For cell lysis, IEC monolayers or intestinal mucosa were harvested in RIPA buffer (0.5\% Triton X-100, 0.5\% NP-40, 0.5\% deoxycholic acid, 0.1\% SDS, $150 \mathrm{mM} \mathrm{NaCl}$, $1 \mathrm{mM}$ EGTA [pH 8.0], $1 \mathrm{mM}$ EDTA, $0.2 \mathrm{mM}$ sodium orthovanadate, $20 \mathrm{mM}$ Tris [pH 7.4]) supplemented with protease and phosphatase inhibitors. Immunoblotting was performed as described previously (7). For immunofluorescence labeling, frozen sections from the colon or IECs grown on permeable supports or glass coverslips were fixed, permeabilized, and incubated with primary and secondary Abs.

Isolation of LP cells. Punch biopsies (2-mm) of wounded or intact colon were taken, and tissue from each experimental condition was placed in $10 \mathrm{ml}$ of RPMI media containing $150 \mu$ liberase stock (2.5 $\mathrm{mg} / \mathrm{ml})$ and $150 \mu \mathrm{l}$ DNase I stock $\left(2 \times 10^{4} \mathrm{Kuntz}\right.$ units $\left./ \mathrm{ml}\right)$ (both from Sigma-Aldrich). Biopsy punches were digested at $37^{\circ} \mathrm{C}$ for 30 minutes and then passed several times through an 18-gauge needle plus a 3-cc syringe, filtered through a $70-\mu \mathrm{m}$ cell strainer into a clean $50-\mathrm{ml}$ tube on ice, and centrifuged to pellet the immune cells. Cells were resuspended in flow cytometry buffer.

Flow cytometry and cell sorting. Isolated LP cells from colonic wounds were resuspended in PBS containing 2\% FBS and then incubated for 30 minutes at $4^{\circ} \mathrm{C}$ with a LIVE/DEAD dye (eBioscience Fixable Viability Dye eFluor 780; Invitrogen, Thermo Fisher Scientific). Cells were washed 2 times in PBS containing 2\% FBS and stained for 30 minutes at $4^{\circ} \mathrm{C}$ with labeled Abs in the presence of Fc block. Samples were then washed 2 times in PBS containing 2\% FBS and analyzed immediately. Flow cytometric analysis was performed on a NovoCyte Flow Cytometer (ACEA Biosciences Inc.). For sorting experiments, isolated LP cells resuspended in PBS containing 2\% FBS were first stained with labeled Abs in the presence of $\mathrm{Fc}$ block for 30 minutes at $4^{\circ} \mathrm{C}$. After washing 2 times in PBS containing 2\% FBS, cells were resuspended in sorting buffer (HBSS containing $10 \mathrm{mM}$ HEPES and 0.1\% FBS) containing LIVE/ DEAD Dye 7-AAD (BD Biosciences) for 20 minutes at $4^{\circ} \mathrm{C}$. Without washing, sorting samples were acquired using a FACSAria II cell sorter at the University of Michigan Flow Cytometry Core. Cells were sorted into 3 groups for $\mathrm{Il1O}$ mRNA expression analysis: a) $\mathrm{CD} 45^{+} \mathrm{CD} 64^{-}$cells; b) $\mathrm{CD} 45^{+} \mathrm{CD} 64^{+} \mathrm{Ly} 6 \mathrm{C}^{-}$cells; and c) $\mathrm{CD} 45^{+} \mathrm{CD} 64^{+} \mathrm{Ly} 6 \mathrm{C}^{+}$cells. The results were plotted and analyzed using FlowJo software.

$P C R$. Total RNA was isolated from SKCO-15 cells using the RNeasy Kit (QIAGEN) with a DNAse I treatment following the manufacturer's protocol. Total RNA ( $1 \mu \mathrm{g})$ was reverse transcribed into cDNA using iScript Reverse Transcription Supermix (Bio-Rad). Samples were evaluated using a PrimePCR Transcription Array (Bio-Rad). Gene expression changes of interest were confirmed by quantitative PCR (qPCR) using SsoAdvanced Universal SYBR Green (Bio-Rad) with a Bio-Rad CTX Cycler measuring SYBR green incorporation for product detection. Reactions were performed in triplicate with 3 biological replicates. The relative expression of NANOG and OCT4 was calculated by the $2-\Delta \Delta \mathrm{Ct}$ method and normalized to the housekeeping gene TATA-box-binding protein (TBP). The primer sequences were as follows: NANOG (forward): 5'-CCTTCTGCGTCACACCATT-3'; NANOG (reverse): 5'-AACTCTCCAACATCCTGAACC-3'; POU5F1 (forward): TGTGTCTATCTACTGTGTCCCA-3'; POU5F1 (reverse): 5'-GTTGGAGGGAAGGTGAAGTTC-3'.

Detection of IL-10 in wounds and immune cells. Total RNA was isolated from equivalent numbers of wounds or immune cells sorted by flow cytometry using the QIAGEN RNeasy Micro Plus Kit according to the manufacturer's instructions. The Bio-Rad iScript cDNA Synthesis Kit was used to synthesize cDNA from $4 \mu$ total RNA. Digital droplet PCR was performed with QX200 ddPCR EvaGreen Supermix, with $1 \mu \mathrm{l}$ cDNA as the template per reaction using the Bio-Rad QX200 AutoDG Droplet Digital PCR. For droplet generation and quantification, PCR was performed using a Bio-Rad C-1000 Touch PCR machine following the manufacturer's cycling conditions. The following primers were used to detect Il10: (forward) 5'-CCCTGGGTGAGAAGCTGAAG; (reverse) 5'-CACTGCCTTGCTCTTATTTTCACA. Data analysis was done using Bio-Rad QuantaSoft software. IL-10 copies/ $\mu$ l were normalized to the Rps18 copies/ $\mu$ f for each sample.

$A b s$ and reagents. The following Abs were used for Western blotting, flow cytometry, and immunofluorescence analysis of structural proteins, signaling molecules, and cell line-specific proteins: IL-10 (catalog 9969) and WISP-1 (catalog 155654) from Abcam; IL-10 
(catalog 12163), GAPDH (catalog D16H11), phosphorylated CREB (p-CREB) (catalog 9198), and CREB (catalog 9197) from Cell Signaling Technology; GBP-1 (catalog sc-53857) from Santa Cruz Biotechnology Inc.; and IL-10R $\alpha$ (catalog PA5-27772) from Thermo Fisher Scientific; FITC-conjugated anti-mouse CD11b (catalog 11-0112-85), eFluor 450-conjugated anti-mouse Ly6C (catalog 48-5932-82) and eFluor 780 LIVE/DEAD stain from eBioscience (catalog 65-0865); PE-conjugated anti-mouse F4/80 (catalog MF48004) from Invitrogen (Thermo Fisher Scientific); PerCP/Cy5.5-conjugated anti-mouse Ly6G (catalog 127616) and PE/Cy7-conjugated anti-mouse CD64 (catalog 139313) from BioLegend; and allophycocyanin-conjugated anti-mouse CD45 (catalog 559864) from BD Biosciences. WISP-1neutralizing Abs AF 1680 (mouse mAb) and AF 1627 (goat polyclonal $\mathrm{Ab}$ ) were purchased from R\&D Systems. The Abs were used at 1:1,000 for Western blotting, 1:100 for immunofluorescence, and 1:200 for flow cytometry. The CREB-CBP interaction inhibitor CAS 92-78-4 was purchased from EMD Millipore and used at a concentration of $10 \mu \mathrm{M}$. IL-10 was used at a concentration of $100 \mathrm{nM}$, and WISP-1 was used at $500 \mathrm{ng} / \mathrm{ml}$. BSA diluted at this concentration was used as a control.

IL-10 and WISP-1 ELISA. IL-10 and WISP-1 ELISA kits were purchased from Abcam (catalogs ab100697 and ab155445, respectively). ELISA assays were performed using cell culture supernatants or tissue lysates derived from punch biopsies obtained from wounded or intact colonic mucosa according to the manufacturer's instructions.

Quantification offecal LCN2 by ELISA. LCN2 levels were quantified in the supernatants of freshly collected fecal samples reconstituted in PBS containing 0.1\% Tween-20 $(100 \mathrm{mg} / \mathrm{ml})$ using the DuoSet Murine LCN2 ELISA Kit (R\&D Systems).

Ex vivo culture of murine mucosal colonic wounds. Small colonic tissue biopsies (4-mm) containing the forceps-induced wounds were removed by punch biopsy. Tissues were rinsed with sterile PBS to remove cellular debris and immediately placed into a 24 -well culture plate (3 wound tissues from each mouse per well). Cultures were maintained for 4 hours at $37^{\circ} \mathrm{C}$ and $5 \% \mathrm{CO}_{2}$ before collection of supernatants.

Generation of the WISP1 promoter. A Homo sapiens WISP1 promoter reporter construct was made in the pGL4.10[luc2] vector (Promega). Genomic DNA was isolated from the SKCO-15 human colonic intestinal cell line using the DNeasy Blood and Tissue Kit (QIAGEN). A portion of human genomic DNA spanning the region from -328 to -39 bp relative to the WISP-1 translation start site was amplified using PCR from $100 \mathrm{ng}$ genomic DNA with a $5^{\prime}$ SacIcontaining (indicated in lowercase letters) sense primer $5^{\prime}$-ctgagctcCACCCTAGTGTGAAGTCATAG and a HindIII containing (lowercase) antisense primer $5^{\prime}$-gattgccaagcttAGAGGATCCGACCACCTC using pFU Ultra polymerase (Agilent Technologies), and then gel purified, digested with SacI/HindIII, and ligated into a SacI/HindIII-digested pGL4.10[luc2] vector. The resulting clones were sequence verified for the correct insertion of the -328 to -39 bp WISP-1 region into the pGL4.10[luc2] vector. A CREB-binding site in the H.s. WISP1 promoter has been previously described (12). Mutation of the CREB-binding site in the pGL4.10[luc2] WISP-1 reporter construct was performed using the QuickChange Kit (Agilent Technologies) with the following primers: sense, 5'-GTCCTTCACCCTGAATTCAGATCTTGCTTTAATA; antisense, 5'-TATTAAAGCAAGATCTGAATTCAGGGTGAAGGAC. The mutation (TGACGTCA -149 to TGAATTCA) was confirmed by nucleotide sequencing. pGL4.10[luc2] served as a vector control.

Luciferase assay. SKCO-15 cells were plated in 48-well plates and transfected with $0.2 \mathrm{mg} /$ well of the pGL4.10 vector containing the WISP1 promoter with an intact or mutated CREB-binding site and 0.04 $\mathrm{mg} /$ well pRL-TK expressing Renilla luciferase (Promega) as a control for transfection efficiency. Samples were generated in triplicate in 48-well tissue culture plates. Luciferase activity was measured, and reporter activity was determined using the Dual Luciferase Reporter Assay System (Promega) according to the manufacturer's protocol.

Study approval. All animal experiments were approved by the IACUCs of the University of Michigan and Emory University and performed according to NIH guidelines.

Statistics. Statistical comparisons were made using either a 2-tailed Student's $t$ test or ANOVA with Tukey's multiple comparisons post test, as appropriate. A $P$ value of less than 0.05 was considered significant.

\section{Author contributions}

$\mathrm{MQ}, \mathrm{HN}$, and PAN designed the study, performed data acquisition, analysis, and interpretation, and wrote the manuscript. MNO, GL, VGH, VA, DS, MF, GB, RH, HW, and CGS performed data acquisition, analysis, and interpretation and edited the manuscript. PHD and JS provided human samples for primary enteroid culture. JCB interpreted results and provided critical review of the manuscript. AN, TLD, and CAP designed the study, supervised the experiments, analyzed and interpreted the data, prepared the manuscript, and obtained funding.

\section{Acknowledgments}

IL-10 $10^{\mathrm{fl} / \mathrm{fl}}$ mice were provided by Werner Müller (Faculty of Life Sciences, University of Manchester, Manchester, United Kingdom). This work was supported by NIH grants (RO1DK055679, RO1DK089763, and DK059888, to AN; R01DK097256, to TLD; and DK61739, DK72564, and DK79392, to CAP); a Crohn's and Colitis Foundation of America Research Fellowship Award (326912, to MQ); and the German Research Foundation (DFG) (NE 1834/1-1, to PAN).

Address correspondence to: Asma Nusrat, 4057 BSRB, 109 Zina Pitcher Place, Ann Arbor, Michigan 48109-2200, USA. Phone: 734.764.5712; Email: anusrat@umich.edu. Or to: Timothy L. Denning, 690 Petit Science Center. 100 Piedmont Avenue SE. Atlanta, Georgia 30303-5090, USA. Phone: 404.413.3609; Email: tdenning@gsu.edu.
1. Wallace KL, Zheng LB, Kanazawa Y, Shih DQ. Immunopathology of inflammatory bowel disease. World J Gastroenterol. 2014;20(1):6-21.

2. Leoni G, Neumann PA, Sumagin R, Denning TL, Nusrat A. Wound repair: role of immune-epithelial interactions. Mucosal Immunol. 2015;8(5):959-968.

3. Kole A, Maloy KJ. Control of intestinal inflamma- tion by interleukin-10. Curr Top Microbiol Immunol. 2014;380:19-38.

4. Kühn R, Löhler J, Rennick D, Rajewsky K, Müller W. Interleukin-10-deficient mice develop chronic enterocolitis. Cell. 1993;75(2):263-274.

5. Denning TL, Wang YC, Patel SR, Williams IR, Pulendran B. Lamina propria macrophages and dendritic cells differentially induce regulatory and interleukin 17-producing $\mathrm{T}$ cell responses. Nat Immunol. 2007;8(10):1086-1094.

6. Berschneider B, Königshoff M. WNT1 inducible signaling pathway protein 1 (WISP1): a novel mediator linking development and disease. Int J Biochem Cell Biol. 2011;43(3):306-309. 
7. Leoni G, et al. Annexin A1, formyl peptide receptor, and NOX1 orchestrate epithelial repair. JClin Invest. 2013;123(1):443-454.

8. Leoni G, et al. Annexin A1-containing extracellular vesicles and polymeric nanoparticles promote epithelial wound repair. J Clin Invest. 2015;125(3):1215-1227.

9. Chassaing B, Srinivasan G, Delgado MA, Young AN, Gewirtz AT, Vijay-Kumar M. Fecal lipocalin 2 , a sensitive and broadly dynamic non-invasive biomarker for intestinal inflammation. PLoS ONE. 2012;7(9):e44328.

10. Ranatunga DC, et al. A protective role for human IL-10-expressing CD4+ T cells in colitis. J Immunol. 2012;189(3):1243-1252.

11. Yanaba K, Yoshizaki A, Asano Y, Kadono T, Tedder TF, Sato S. IL-10-producing regulatory B10 cells inhibit intestinal injury in a mouse model. Am J Pathol. 2011;178(2):735-743.

12. Xu L, Corcoran RB, Welsh JW, Pennica D, Levine AJ. WISP-1 is a Wnt-1- and beta-catenin-responsive oncogene. Genes Dev. 2000;14(5):585-595.

13. Capaldo CT, et al. IFN- $\gamma$ and TNF- $\alpha$-induced GBP-1 inhibits epithelial cell proliferation through suppression of $\beta$-catenin/TCF signaling. Mucosal Immunol. 2012;5(6):681-690.

14. Sansom OJ, et al. Myc deletion rescues Apc deficiency in the small intestine. Nature. 2007;446(7136):676-679.

15. Königshoff M, et al. WNT1-inducible signaling protein-1 mediates pulmonary fibrosis in mice and is upregulated in humans with idiopathic pulmonary fibrosis. JClin Invest. 2009;119(4):772-787.

16. Blander JM. Death in the intestinal epitheliumbasic biology and implications for inflammatory bowel disease. FEBS J. 2016;283(14):2720-2730.

17. Rivollier A, He J, Kole A, Valatas V, Kelsall BL. Inflammation switches the differentiation program of Ly6Chi monocytes from antiinflammatory macrophages to inflammatory dendritic cells in the colon. J Exp Med. 2012;209(1):139-155.

18. Hayashi A, et al. A single strain of Clostridium butyricum induces intestinal IL-10-producing macrophages to suppress acute experimental colitis in mice. Cell Host Microbe. 2013;13(6):711-722.

19. Ueda Y, et al. Commensal microbiota induce LPS hyporesponsiveness in colonic macrophages via the production of IL-10. Int Immunol. 2010;22(12):953-962.

20. Novak ML, Weinheimer-Haus EM, Koh TJ. Macrophage activation and skeletal muscle healing following traumatic injury. J Pathol. 2014;232(3):344-355.

21. Verma SK, et al. IL-10 Accelerates ReEndothelialization and Inhibits Post-Injury Intimal Hyperplasia following Carotid Artery Denudation. PLOS ONE. 2016;11(1):e0147615. 22. Inoue G. Effect of interleukin-10 (IL-10) on experimental LPS-induced acute lung injury. J Infect Chemother. 2000;6(1):51-60.

23. Kominsky DJ, et al. IFN- $\gamma$-mediated induction of an apical IL-10 receptor on polarized intestinal epithelia. J Immunol. 2014;192(3):1267-1276.

24. King A, Balaji S, Le LD, Marsh E, Crombleholme TM, Keswani SG. Interleukin-10 regulates fetal extracellular matrix hyaluronan production. JPediatr Surg. 2013;48(6):1211-1217.

25. Balaji S, et al. The role of interleukin-10 and hyaluronan in murine fetal fibroblast function in vitro: implications for recapitulating fetal regenerative wound healing. PLOS ONE. 2015;10(5):e0124302.

26. Autschbach F, et al. In situ expression of interleukin10 in noninflamed human gut and in inflammatory bowel disease. Am JPathol.1998;153(1):121-130.

27. Jarry A, et al. Mucosal IL-10 and TGF-beta play crucial roles in preventing LPS-driven, IFN-gamma-mediated epithelial damage in human colon explants. J Clin Invest. 2008;118(3):1132-1142.

28. Kasten KR, Muenzer JT, Caldwell CC. Neutrophils are significant producers of IL-10 during sepsis. Biochem Biophys Res Commun. 2010;393(1):28-31.

29. Goto Y, et al. IL-10-producing CD4(+) T cells negatively regulate fucosylation of epithelial cells in the gut. Sci Rep. 2015;5:15918.

30. Leibovich SJ, Ross R. The role of the macrophage in wound repair. A study with hydrocortisone and antimacrophage serum. Am J Pathol. 1975;78(1):71-100.

31. Hunt TK, Knighton DR, Thakral KK, Goodson WH, Andrews WS. Studies on inflammation and wound healing: angiogenesis and collagen synthesis stimulated in vivo by resident and activated wound macrophages. Surgery. 1984;96(1):48-54.

32. Mirza R, DiPietro LA, Koh TJ. Selective and specific macrophage ablation is detrimental to wound healing in mice. Am J Pathol. 2009;175(6):2454-2462.

33. Lucas $\mathrm{T}$, et al. Differential roles of macrophages in diverse phases of skin repair. JImmunol. 2010;184(7):3964-3977.

34. Bain CC, et al. Constant replenishment from circulating monocytes maintains the macrophage pool in the intestine of adult mice. Nat Immunol. 2014;15(10):929-937.

35. Itoh K, Hirohata S. The role of IL-10 in human B cell activation, proliferation, and differentiation. J Immunol. 1995;154(9):4341-4350.

36. Thompson-Snipes L, Dhar V, Bond MW, Mosmann TR, Moore KW, Rennick DM. Interleukin 10: a novel stimulatory factor for mast cells and their progenitors. J Exp Med. 1991;173(2):507-510.

37. Dosanjh A, Morris RE, Wan B. Bronchial epithelial cell-derived cytokine IL-10 and lung fibroblast proliferation. Transplant Proc. 2001;33(1-2):352-354.

38. Eming SA, et al. Accelerated wound closure in mice deficient for interleukin-10. Am J Pathol. 2007;170(1):188-202.

39. Shaywitz AJ, Greenberg ME. CREB: a stimulusinduced transcription factor activated by a diverse array of extracellular signals. Annu Rev Biochem. 1999;68:821-861.

40. Wen AY, Sakamoto KM, Miller LS. The role of the transcription factor CREB in immune function. JImmunol. 2010;185(11):6413-6419.

41. Della Fazia MA, Servillo G, Sassone-Corsi P. Cyclic AMP signalling and cellular proliferation: regulation of CREB and CREM. FEBS Lett. 1997;410(1):22-24.

42. Dworkin S, et al. CREB activity modulates neura cell proliferation, midbrain-hindbrain organization and patterning in zebrafish. Dev Biol. 2007;307(1):127-141.

43. Bird AD, et al. cAMP response element binding protein is required for differentiation of respiratory epithelium during murine development. PLOS ONE. 2011;6(3):e17843.

44. Xu J, Tian J, Grumelli SM, Haley KJ, Shapiro SD. Stage-specific effects of cAMP signaling during distal lung epithelial development. J Biol Chem. 2006;281(50):38894-38904

45. Gubbay O, Rae MT, McNeilly AS, Donadeu FX, Zeleznik AJ, Hillier SG. cAMP response element-binding (CREB) signalling and ovarian surface epithelial cell survival. JEndocrinol. 2006;191(1):275-285

46. Fullerton JN, Gilroy DW. Resolution of inflammation: a new therapeutic frontier. Nat Rev Drug Discov. 2016;15(8):551-567.

47. Kucharzik T, Stoll R, Lügering N, Domschke W. Circulating antiinflammatory cytokine IL-10 in patients with inflammatory bowel disease (IBD) Clin Exp Immunol. 1995;100(3):452-456.

48. [No authors listed]. Seventh international workshop on the CCN family of genes. JCell Commun Signal. 2014;8(1):77-92.

49. Loh YH, et al. The Oct4 and Nanog transcription network regulates pluripotency in mouse embryonic stem cells. Nat Genet. 2006;38(4):431-440.

50. Li J, Li J, Chen B. Oct4 was a novel target of Wnt signaling pathway. Mol Cell Biochem. 2012;362(12):233-240.

51. Klee S, Lehmann M, Wagner DE, Baarsma HA, Königshoff M. WISP1 mediates IL-6-dependent proliferation in primary human lung fibroblasts. Sci Rep. 2016;6:20547.

52. Roers A, et al. T cell-specific inactivation of the interleukin 10 gene in mice results in enhanced $\mathrm{T}$ cell responses but normal innate responses to lipopolysaccharide or skin irritation. J Exp Med. 2004;200(10):1289-1297. 